\title{
Arbor
}

\section{La Enseñanza Naval}

\author{
Emilio J. Nieto Manso
}

Arbor CLXXIII, 682 (Octubre 2002), 251-261 pp.

La situación mundial en los albores del siglo XXI presenta un escenario de drásticos y rápidos cambios que, naturalmente, afectan también a los ámbitos de Seguridad y Defensa. La Armada, consciente de que su centro de gravedad reside en la calidad humana y competencia profesional de los hombres y mujeres que la componen, se enfrenta al futuro con el decidido propósito de potenciar su enseñanza y formación.

El Director de Enseñanza Naval, tras una breve descripción de los diferentes niveles de enseñanza y centros docentes con que cuenta actualmente la Armada, centra su artículo en un acertado análisis de los factores determinantes que han conducido a un proceso de reestructuración para racionalizar los medios disponibles y mejorar la calidad de la enseñanza, finalizando su presentación con una síntesis sobre las principales lineas de actuación a corto y medio plazo, y la implantación progresiva de un ambicioso Plan de Innovación Tecnológica de la Enseñanza en la Armada.

«En buena o mala fortuna la Milicia no es más que una Religión de Hombres honrados». Calderón de la Barca

La situación mundial en los inicios del Siglo XXI presenta un escenario de drásticos y rápidos cambios que en el campo de la Seguridad y Defensa se materializan en: la ausencia de la confrontación de bloques, motivada por la desaparición del Pacto de Varsovia, consecuencia del derrumbamiento del sistema marxista; la nueva estrategia de diálogo y cooperación en la Alianza Atlántica, que estrecha las relaciones entre 
los que en su día fueron adversarios; la sustitución de las «amenazas» por «riesgos» de diferente naturaleza y procedencia multipolar; y el desarrollo de capacidades de Defensa de carácter colectivo en el ámbito de diferentes Organizaciones Internacionales (ONU, OSCE, OTAN, UEO, UE, etc.)

Coincidiendo con estos cambios se ha llevado a cabo el proceso de profesionalización de nuestras Fuerzas Armadas, culminado en un corto espacio de tiempo, con una evolución tecnológica en proceso muy acelerado y una falta de conciencia de la sociedad en relación con la Defensa, lo que dificulta la captación del personal necesario en cantidad y calidad.

Ante esta situación, la Armada, que siempre ha tenido conciencia clara de que su valor es el de los hombres y mujeres que la componen, se enfrenta al futuro, una vez más, con el decidido propósito de superar todo tipo de dificultades; y la enseñanza y formación de su gente se constituye en factor primordial para alcanzar el objetivo de dotarse de personal debidamente preparado para desarrollar sus capacidades, en los ámbitos conjunto e internacional, obteniendo el máximo rendimiento de la Fuerza disponible.

\section{Introducción}

La Enseñanza en la Armada está encuadrada dentro del Departamento de Personal, en la Dirección de Enseñanza Naval (DIENA), cuyo director es un Contralmirante del Cuerpo General (ADIENA).

La Enseñanza Naval forma parte de la Enseñanza General Militar -concebida de acuerdo con los principios constitucionales y como fundamento del ejercicio profesional en las Fuerzas Armadas-, y tiene como objetivo la formación integral y la capacitación específica del militar, así como la permanente actualización de sus conocimientos en los ámbitos operativo, científico, técnico y de gestión de recursos. Se configura como un sistema unitario que garantiza la continuidad del proceso educativo en la Armada, e integrado en el sistema educativo nacional.

La Enseñanza Militar se estructura en:

- Enseñanza Militar de Formación.

- Enseñanza Militar de Perfeccionamiento.

- Altos Estudios Militares.

La Enseñanza Militar de Formación tiene como finalidades la preparación para la incorporación a las Escalas de Militares de Carrera 


\section{La Enseñanza Naval}

y la capacitación para el acceso a Militar de Complemento y a Militar de Tropa y Marinería.

Para la incorporación a las Escalas de Militares de Carrera, la Enseñanza Militar de Formación presenta tres niveles:

- Enseñanza para la incorporación a las Escalas de Suboficiales, que se corresponde con la Formación Profesional de Grado Superior.

- Enseñanza para la incorporación a las Escalas de Oficiales, que se corresponde con la Educación Universitaria de Primer Ciclo, y

- Enseñanza para la incorporación a las Escalas Superiores de Oficiales, que se corresponde con la Educación Universitaria de Segundo Ciclo.

La Enseñanza Militar de Perfeccionamiento va dirigida a capacitar al militar para el desempeño de los cometidos en empleos superiores y a proporcionarle los conocimientos correspondientes a las Especialidades Complementarias; es decir, ampliar o actualizar los conocimientos requeridos en un aspecto específico, necesario para el desarrollo de la profesión militar en un determinado ámbito.

Por último, la Enseñanza de Altos Estudios Militares abarca la preparación del Militar de Carrera de la Escala Superior de Oficiales para el desarrollo de actividades en los Estados Mayores, y la capacitación para el desempeño de los cometidos en los empleos de General de Brigada o Contralmirante. También se consideran Altos Estudios Militares aquéllos que están relacionados con la Paz y la Seguridad, la Defensa Nacional y la Política Militar, así como con la investigación y desarrollo de las doctrinas para el empleo de las Fuerzas Armadas.

\section{Centros de enseñanza}

Actualmente la Armada dispone de diecisiete centros docentes, distribuidos geográficamente en Galicia (4), Cartagena (4), Bahía de Cádiz (5) y Madrid (4).

En Galicia:

Escuela Naval Militar (ENM) en Marín, Escuela de Transmisiones y Electrónica (ETEA) en Vigo, Escuela de Especialidades «Antonio de Escaño» en Ferrol y Grupo de Escuelas de la Estación Naval de La Graña, también en Ferrol. 


\section{En Cartagena:}

Escuela de Formación de Tropa de Infantería de Marina (ESFORTIM), Escuela de Submarinos, Escuela de Especialidades «Antonio de Ulloa» y Centro de Buceo de la Armada (CBA).

En la Bahía de Cádiz:

Escuela de Dotaciones Aeronavales (EDAN) en la Base Naval de Rota, Escuela de Suboficiales (ESUBO) en San Fernando, Escuela de Infantería de Marina (EIM) en San Fernando, Escuela de Estudios Superiores en San Fernando e Instituto Hidrográfico en Cádiz.

En Madrid:

Escuela de Guerra Naval (EGN), Centro de Estudios Superiores de Intendencia (CESIA), Escuela Técnica Superior de Ingenieros de Armas Navales (ETSIAN) y Escuela de Informática.

El único centro docente de la Armada dedicado exclusivamente a la Enseñanza de Formación es la Escuela Naval Militar. Los demás centros comparten dos o los tres tipos de enseñanza, lo que se explica por la necesidad de racionalización de recursos e infraestructura. Esta circunstancia hace muy difícil la clasificación de los centros atendiendo al tipo de enseñanza que imparten.

Otra forma de clasificar los Centros de Enseñanza en «Academias», «Escuelas» y «Unidades-Escuela», puede proporcionar una visión más clara de los medios disponibles:

En la Escuela Naval Militar se lleva a cabo la formación «académica» de todos los Oficiales Superiores y Oficiales de la Armada, en cualquiera de las modalidades de acceso. En unos casos la formación que se imparte es total y en otros se atiende en exclusiva a la Formación General Militar, que se completa luego en las Escuelas de Especialidades Fundamentales (Ingenieros o Cuerpos de Especialistas).

En la Escuela de Suboficiales se imparte la Formación General Militar para el acceso a la Escala de Suboficiales del Cuerpo de Especialistas, formación que se completa a continuación en las Escuelas de Especialidades Fundamentales. En este centro se imparte asimismo el curso de capacitación para el ascenso a Suboficial Mayor y a Cabo Mayor.

En las Escuelas de Especialidades Fundamentales se capacita a los Suboficiales, una vez adquirida la formación militar común y básica, para el desempeño de una determinada función (Comunicaciones, Electrónica, Administración, etc...). Estas Escuelas son: 


\section{La Enseñanza Naval}

- Comunicaciones y Electrónica ETEA (Vigo

- Energía y Propulsión ANTONIO DE ESCAÑO (Ferrol).

- Administración, Maniobra y Hostelería LA GRAÑA (Ferrol).

- Armas ANTONIO DE ULLOA (Cartagena)

- Actividades Subacuáticas C B A (Cartagena).

- Hidrografía INSTITUTO HIDROGRAFICO (Cádiz)

En estas Escuelas, especializadas en unos determinados currículos, también se proporciona la formación completa de la Marinería y Tropa de acuerdo con sus Especialidades, así como las enseñanzas correspondientes a las Especialidades Complementarias de los Oficiales, Cursos Monográficos y de ascenso de Marinería y Tropa.

En las Unidades-Escuela se imparte la enseñanza correspondiente a determinadas Aptitudes o Especialidades Complementarias (Submarinos, Aeronaves, étc.) o de Formación de Tropa de Infantería de Marina (ESFORTIM).

Un desarrollo estricto de todas las posibilidades que contempla la Ley 17/99 (Acceso Directo, Promoción Interna y Cambio de Cuerpo) significa para la Armada la elaboración de 51 Planes de Estudio para Acceso Directo, 35 para Promoción Interna y 25 para Cambio de Cuerpo, además de los que, en su día, puedan corresponder a la formación de reservistas. Si al más del centenar de cursos resultantes se les añaden los de Especialidades Complementarias y hasta 300 cursos monográficos para obtener cualificaciones en un determinado equipo o sistema, tendremos una idea de la carga actual de trabajo de los centros docentes y la complejidad que implica su control y planificación por la Dirección de Enseñanza Naval.

\section{Reestructuracion de centros}

El elevado número de Centros de Enseñanza en la Armada y su localización dispersa, respondía a las necesidades y circunstancias de hace cincuenta años, en los que el personal de reemplazo prestaba su Servicio Militar por un período de dos años, que incluía su permanencia en las Escuelas durante seis meses para obtener una formación especializada (Teletipistas, Señaleros, Operadores de Radar, etc.).

Las sucesivas reducciones del Servicio Militar y el proceso de plena profesionalización ya culminado, han ido haciendo progresivamente y cada vez más necesaria una reestructuración que racionalice los medios disponibles y mejore la calidad de la enseñanza impartida. El proceso de 
reestructuración, todavía en curso, se ha iniciado con las siguientes actuaciones:

- Integración del antiguo Centro de Especialistas de Infantería de Marina en el Tercio de Levante, creándose la ESFORTIM como Unidad-Escuela, de forma similar a las Escuelas de Submarinos, Dotaciones Aeronavales, etc.

- A lo largo del año en curso las enseñanzas que se imparten en la ETEA se integrarán en la Escuela «Antonio de Escaño», procediéndose al cierre de las instalaciones en Vigo.

- Se encuentran en estudio otros proyectos de racionalización.

\section{Factores que afectan a la planificación}

Dentro del marco general de actuación definido por la Ley y con la estructura de centros docentes antes señalada, existen una serie de factores que afectan a la planificación de la Enseñanza Naval y que son básicamente los siguientes:

- Incorporación de España a diversos Organismos Internacionales.

- Adaptación a las nuevas tecnologías.

- Plena profesionalización del personal.

- Modelo de selección continuada.

- Adaptación de la enseñanza a los nuevos requisitos de la Fuerza.

- Elevado número de cursos.

- Nuevos procedimientos de trabajo.

- Calidad de la enseñanza.

- Necesidad de Ingenieros de Armas Navales.

La incorporación de España a diversos Organismos Internacionales obliga a un numeroso despliegue de personal de la Armada en el extranjero con la exigencia de un conocimiento de idiomas, en particular del inglés, a nivel de "posee» e incluso superior. Esta circunstancia obliga a mejorar a todos los niveles el grado de conocimiento, que es particularmente bajo a nivel de Suboficiales (sólo el 2\% tiene el «posee») y prácticamente nulo a nivel de Marinería y Tropa. Por otra parte, también resulta necesario elevar el nivel de conocimiento sobre las citadas Organizaciones y de sus procedimientos de trabajo.

La adaptación a nuevos entornos tecnológicos, existentes tanto en el ámbito civil como en el militar, obliga a abordar este cambio con el empleo de nuevas tecnologías e impulsar la superación de barreras culturales que impiden acceder con confianza a las nuevas «herramientas». 


\section{La Enseñanza Naval}

La culminación del proceso de profesionalización de Marinería y Tropa hace que la primera prioridad de la Armada sea el personal, y que para su «motivación» sea absolutamente necesaria una oferta de futuro atractiva, cuya base será sin duda la calidad y actualidad de la formación, lo que obliga a cuidar de forma muy especial los medios que se ponen a su disposición en las Escuelas, no sólo en lo que se refiere a la idoneidad del personal docente, sino también en otros aspectos como la habitabilidad, ayudas a la enseñanza, asistencia a las familias, etc. Se trata de pasar a una enseñanza rica en aprendizaje, en la que el centro de gravedad se encuentra en el alumno.

La reducción de efectivos de Marinería y Tropa, consecuencia de la profesionalización y la necesidad de destinarlos a las Unidades Operativas para mantener una "masa crítica» en la Fuerza, razón de ser de la Armada, disminuye el personal disponible en los Centros de Enseñanza, lo que refuerza la estrategia adoptada de reestructurar la disposición y número de centros existentes.

El modelo de selección continuada de personal de Marinería y Tropa es otro importante factor a tener presente en la programación de la enseñanza. Si en unas épocas se incrementa la captación, quedará afectada la capacidad de las Escuelas (alojamientos, aulas, profesorado) y por lo tanto la calidad de la formación del alumno. Por el contrario, si la captación es escasa, los cursos no resultarán rentables. El equilibrio debe alcanzarse precisamente con una selección continuada, fijando un número adecuado de incorporaciones a las Escuelas, que se estima podría ser de cinco convocatorias al año.

Por otra parte, la próxima incorporación a la Fuerza de las Fragatas F-100, con sistemas vanguardistas como el sistema AEGIS, obliga no sólo a un esfuerzo inicial considerable para formar las primeras dotaciones, sino también a una mejora en la formación específica de este personal, acostumbrándolo desde el principio al empleo de tecnologías-punta, así como a un mayor tiempo de permanencia del personal en dichos buques.

La dotación económica que la Armada ha dedicado a la Enseñanza de Perfeccionamiento y Altos Estudios Militares ha crecido progresivamente en los últimos años, llegando a suponer en la actualidad el 2.2\% del total del presupuesto de la Armada. Esta tendencia creciente es consecuencia del incremento progresivo de la tasa de profesionalización y de la puesta en marcha de los programas de nuevas construcciones. Esta circunstancia obliga a revisar el modelo actual de formación para economizar recursos. Es posible a medio plazo una reducción del gasto aplicando nuevas tecnologías, pero ello obliga a generalizar el conocimiento de la informática para usuarios a todos los niveles. 
No existe en la actualidad un instrumento fiable que nos permita medir la calidad de la enseñanza impartida en los centros docentes de la Armada; aunque los resultados pueden considerarse buenos, se estima que son mejorables.

Por último, la Armada se encuentra necesitada de Ingenieros de Armas Navales. El sistema de acceso directo con titulación previa no satisface estas necesidades, ya que la Universidad no proporciona esta titulación y el tiempo establecido para su formación en los centros militares no es suficiente para proporcionar a los alumnos la necesaria Formación General Militar y la específica en su rama.

\section{Planes de actuacion a corto plazo}

Dentro del marco que define la Ley 17/99, la Armada ha iniciado las acciones necesarias para dar debida respuesta a la problemática descrita en el punto anterior, del siguiente modo:

En lo que respecta a la enseñanza del idioma inglés, se han establecido unos niveles mínimos de conocimientos para acceder a los Centros de Enseñanza de Formación, niveles que se van elevando posteriormente durante el desarrollo de los períodos de formación y perfeccionamiento. Se han creado Departamentos de Idiomas en las Escuelas, desapareciendo las anteriores Escuelas de Idiomas de Zona. Por otra parte, se ha incrementado el número de horas lectivas en los programas y se está dotando a las distintas Unidades y Dependencias de la Armada de cursos interactivos y medios informáticos adicionales para el estudio de idiomas.

Se están ordenando los Planes de Estudio de la Enseñanza de Formación para dar debida respuesta a las necesidades de conocimiento, a todos los niveles, de los Organismos Internacionales en los que participa la Armada.

La adaptación a las nuevas tecnologías se ha emprendido a través de la potenciación de las Escuelas mediante la instalación de aulas multimedia conectadas a la Intranet de la Armada, la potenciación del Centro de Ayudas a la Enseñanza (CAE) y su capacitación como creador de productos multimedia, así como la aplicación de técnicas de Gestión del Conocimiento a la gestión y desarrollo de la Dirección de Enseñanza Naval. La mayor parte de estas acciones, están recogidas en los Planes de actuación a Medio Plazo.

Como desarrollo de la Ley 17/99, está previsto proporcionar en un futuro inmediato al personal de Marinería y Tropa la homologación de los estudios que realicen en los Centros Militares de Enseñanza, 
con un título del Sistema Educativo General (Técnico en Defensa) mediante el incremento correspondiente de horas lectivas (cifrado en 1300), manteniéndose el plań de formación específico para atender a las necesidades operativas de la Fuerza. Al mismo tiempo, el Ministerio de Defensa estudia la convalidación de los estudios de Especialidad específicos por un título del Sistema Educativo General (Técnico Militar en ...).

En lo que se refiere a las necesidades de las nuevas Fragatas se estudia la permanencia del personal asignado a estos buques en un ciclo rotativo de 11 años, que incluye y alterna su permanencia a bordo con destinos en Centros de Mantenimiento y en Escuelas.

Se ha reducido el número de alumnos en la Enseñanza de Perfeccionamiento mediante:

- Mejora de la progresividad en la enseñanza, reduciendo al mínimo la repetición de materias e incrementando la enseñanza por correspondencia.

- Pasando a la Enseñanza de Formación parte de la impartida en Cursos Monográficos.

- Suprimiendo aquellos Cursos Monográficos cuyo conocimiento puede lograrse por un esfuerzo personal del alumno si posee un adecuado nivel de formación previo.

En el campo de la informática se han incrementado los períodos lectivos en los cursos de ascenso a Suboficial, Cabo Primero y Cabo, así como el número de cursos para usuarios tanto en la Escuela de Informática como en los Centros de Apoyo Informático de las Bases y Zonas Marítimas.

Está en proceso de evaluación un procedimiento que permita conocer y valorar la calidad de la Enseñanza de Formación y de Perfeccionamiento impartidas. El procedimiento está basado en lo establecido en la LOGSE (Plan Nacional de Evaluación de la Calidad de Enseñanza en las Universidades de 1997), con aportaciones de lo regulado en otros países de nuestro entorno y del mundo empresarial, y atención especial al modelo seguido por la Marina de Holanda en cuya Escuela Naval se está desarrollando un programa piloto desde hace cuatro años.

Por otra parte, existen en la actualidad catorce Convenios o Acuerdos de colaboración con diversas Universidades, Centros de Investigación y Fundaciones, que inciden en la mejora de la calidad de la enseñanza en los centros docentes de la Armada.

Por último, y en lo que se refiere a la necesidad de formación de Ingenieros de Armas Navales, se pretende facilitar el Cambio de Cuerpo a Oficiales del Cuerpo General e Infantería de Marina, manteniendo además el sistema actual de acceso directo con titulación previa. 


\section{Planes de actuación a medio plazo}

Las necesidades ya apuntadas y derivadas de la incorporación a un marco internacional, la profesionalización de las Fuerzas Armadas, el acceso continuado a la formación, la incorporación de nuevos buques, las restricciones presupuestarias y de personal,...etc., se producen en un entorno civil inmerso en un profundo cambio tecnológico que también se hace patente en el mundo militar.

En este escenario, al que la Armada tiene que incorporarse necesariamente, se considera que el campo de la enseñanza es el lugar idóneo para introducir los nuevos sistemas de gestión y aplicar las nuevas tecnologías. Es en las Escuelas y demás centros docentes donde nuestro personal tiene su primer contacto obligado con la Armada, y donde los modos de actuar y sentir que se adquieran van a tener una importancia capital en el ejercicio de su profesión.

Para ello se ha establecido el Plan de Innovación Tecnológica de la Enseñanza en la Armada (PITEA), desarrollado por el CAE, bajo la dirección de ADIENA, que precisamente tiene como por objetivo promover la modernización de la Enseñanza en la Armada; y para ello comienza proponiendo un nuevo modelo de enseñanza que sirva como vehículo integrador de capacidades, procesos de trabajo y tecnologías. El modelo seleccionado es el titulado «Enseñanza Distribuida», con el que se pretende:

- Proporcionar un entorno adecuado a la enseñanza, para que sea accesible desde cualquier lugar, en cualquier momento y por la persona adecuada.

- Proporcionar formación continuada, relacionando alumnos y materias, según las necesidades, pasando de un concepto de conocimiento estático a uno dinámico.

- Proporcionar información actualizada y potentes bases de datos para que, con el empleo de las herramientas adecuadas, la estructura docente disponga del necesario apoyo para impartir la enseñanza y la oportuna ayuda para la toma de decisiones.

Para conseguir la implantación de este modelo, el PITEA contempla tres planes parciales que desde el primer momento se acometen de forma simultánea, ya que se es consciente de que un desarrollo secuencial no permitiría incorporarse a un mundo tan cambiante como el que se pretende abordar. Estos planes parciales son:

- Potenciar el Centro de Ayudas a la Enseñanza (CAE).

- Potenciar las Escuelas y centros docentes de la Armada

- Potenciar la DIENA a través de la Gestión del Conocimiento. 


\section{La Enseñanza Naval}

Los dos primeros permiten incorporar las infraestructuras tecnológicas necesarias para generar y distribuir el nuevo modelo de enseñanza: y el tercero, introduciendo el concepto de Gestión del Conocimiento, es en el que se concreta, se hace realidad e impulsa el verdadero y profundo cambio que propone el PITEA, al conferir a la DIENA el carácter de Universidad Corporativa Virtual y favorecer una gestión ágil y dinámica de la «Enseñanza Distribuida».

Con ello se espera lograr:

- Reducción del tiempo de enseñanza presencial.

- Potenciación del uso de las TIC.

- Favorecer la profesionalización y modernización de la Armada.

- Formación continuada. Autoaprendizaje centrado en el alumno.

- Favorecer la «motivación» al implicar al usuario directamente en su formación.

- Mejor control del proceso de la Enseñanza Naval, al poder conocer y controlar los accesos a la información y almacenar datos de evaluaciones.

- Reducción de necesidades de profesorado permanente.

- Reducción de costes.

El proyecto PITEA tiene como objeto demostrar en un plazo de 8 - 9 meses las ventajas que podría aportar a la Armada la «Enseñanza Distribuida» y su viabilidad. Para ello se implementará el modelo conceptual que se está definiendo en un centro piloto en la Escuela Naval Militar y, una vez probado y aceptado el modelo, se procedería a su extensión al resto de las Escuelas.

Todas estas medidas a corto y medio plazo deben desembocar en el logro de la calidad de enseñanza debida, para los casi diez mil hombres y mujeres de la Armada, cuya formación anualmente controla la Dirección de Enseñanza Naval. 